\title{
FACETS of health disparities in epilepsy surgery and gaps that need to be addressed
}

Cody L. Nathan, MD*, and Camilo Gutierrez, MD*

Neurology: Clinical Practice August 2018 vol. 8 no. 4 340-345 doi:10.1212/CPJ.0000000000000490
Correspondence

Dr. Gutierrez

CamiloGutierrez@

som.umaryland.edu

\begin{abstract}
Purpose of review

Disparities in treatment and outcomes of patients with epilepsy have been identified in several distinct patient populations. The purpose of this review is to organize the literature and establish clear pathways as to why certain patient populations are not receiving epilepsy surgery. By establishing the acronym FACETS (fear of treatment, access to care, communication barriers, education, trust between patient and physician, and social support), we set up a pathway to further study this area in an organized fashion, hopefully leading to objective solutions.
\end{abstract}
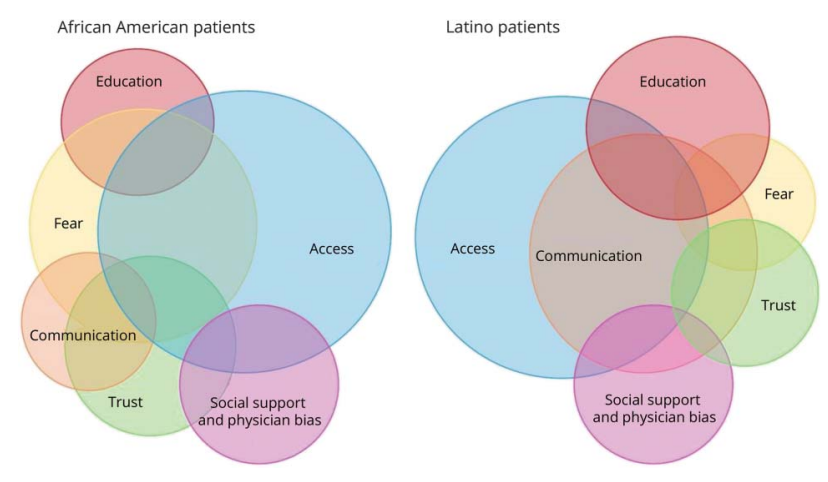

\section{Recent findings}

Studies revealed that African American, Hispanic, and non-English-speaking patients underwent surgical treatment for epilepsy at rates significantly lower compared to white patients.

\section{Summary}

This article explains possible reasons outlined by FACETS for the health disparities in epilepsy surgery that exist in patients of a certain race, socioeconomic status, and language proficiency.

The Centers for Disease Control and Prevention defines health disparities as "preventable differences in the burden of disease, injury, violence, or opportunities to achieve optimal health that are experienced by socially disadvantaged populations." ${ }^{1}$ Populations that experience discrepancies in care include but are not limited to African Americans, Hispanics, Non-English speakers, and patients of low socioeconomic status.

Epilepsy disproportionately affects nonwhite individuals. In 1990, the rate of epilepsy was 1.3-2.2 times greater for nonwhite compared to white male individuals and 1.4-1.7 times greater for nonwhite compared to white female individuals. ${ }^{2}$ Between 1986 and 1990, the ageadjusted prevalence of epilepsy for African Americans was 6.7 per 1,000 compared to whites at 4.5 per $1,000 .^{3}$ Elderly Hispanic men had prevalence rates of $15-18$ per 1,000 compared to non-Hispanic men of a similar age group, with $12-16$ per $1,000 .^{4}$

In terms of treatment, African American and Hispanic patients are less likely to undergo surgical treatment, more likely to be treated in the emergency department with seizures, less likely to receive antiepileptic drugs (AEDs), and less likely to receive specialized care compared to white patients. ${ }^{5-7}$ 
Populations that experience discrepancies in care include but are

not limited to African Americans, Hispanics, Non-English speakers, and patients of low socioeconomic status.

Understanding patient- and provider-related potential causes for the disparity in surgical treatment of epilepsy can be helpful in elucidating factors that contribute to overall health disparities, ultimately leading to interventions and solutions. We have organized the key factors that contribute to surgical disparities with the acronym FACETS, which stands for fear of treatment, access to care, communication barriers, education, trust between patient and physician, and social support. Each of these factors may hold a different weight depending on the race, ethnicity, and culture of the patient (figure). These factors can be decomposed and studied with established metrics and possible interventions can be formulated (table).

\section{Fear of treatment}

Few studies have directly assessed fear with regards to epilepsy surgery. A focus group study out of UCLA noted 6 African American participants expressed "a great deal of fear" with regards to epilepsy surgery. ${ }^{8}$ Specifically, they were afraid of negative outcomes from surgery, such as wordfinding difficulty. Only 3 of these participants said they would consider surgery as an option for treatment even if deemed eligible candidates. African American patients also expressed a sense of worry related to AED side effects.

Though fear of surgery has not been explicitly studied in epilepsy patients, we can learn from studies in other fields of medicine. Compared to white patients, African American patients undergoing arthroplasty surgery had a greater fear score and worse score on the Pain and Anxiety Symptoms Scale both before and after surgery. ${ }^{9}$ Researchers found that guilt and fear were the predominant emotions expressed by African American patients with end stage renal disease who want living donor kidney transplant but did not ask for one. ${ }^{10}$

Fear of treatment likely has complex historical, social, and cultural roots, yet it is an understudied factor related to epilepsy care that can potentially be a major influential factor in a patient's decision for treatment depending on the patient's race/cultural background.

\section{Access to care}

Access encompasses a variety of resources, including access to comprehensive health care, specialized epilepsy centers, and AEDs. Between 1990 and 2008, 6.5\% of white patients with medically refractory epilepsy underwent temporal lobectomy during hospitalization, while rates among racial minorities were significantly less. ${ }^{11}$ Black, Hispanic, Asian/ Pacific Islander, and Native American patients underwent surgery at rates of $2.4 \%-5.9 \%$, with white patients more likely to have private insurance compared to nonwhite patients. Similarly, McClelland et al. ${ }^{12}$ found that having private insurance increased the odds of a patient undergoing anterior temporal lobectomy. The Affordable Care Act (ACA) may have ramifications on the issue of insurance as data from a 2012 to 2013 analysis of patients registered in the National Inpatient Sample showed no racial disparities in access to surgical care for drug-resistant temporal lobe epilepsy since introduction of the ACA. It is worth noting there was no difference in care or outcomes between Caucasian and African American patients, though the sample size was limited to only 11 African American patients who underwent surgery. ${ }^{13}$ Access to specialists is also a major issue when it comes to equal treatment. Compared to white patients, African American and Hispanic patients in New York and Houston had significantly higher rates of generalist visits (odds ratio 5.3 and 4.9) and lower rates of specialist visits (odds ratio 0.3 and 0.4 ). ${ }^{14}$ In addition, patients with lower incomes and those who were uninsured or on Medicare or Medicaid had higher rates of generalist visits, emergency room care, and hospitalizations plus lower rates of specialist visits compared to patients with higher incomes and private insurance. This discrepancy was still significant even after adjusting for age, sex, seizure frequency, and comorbid diseases, verifying that disease burden did not explain the difference in care. African Americans are also more likely to have nonprivate insurance, less likely to have evidence of regular ambulatory care, and more likely to have multiple visits within the calendar year compared to white patients. ${ }^{15}$ These findings were reproduced and expanded upon by Schiltz et al., ${ }^{7}$ who analyzed 115,632 patients with persistent seizures, revealing 9\% of African American and 24\% of Hispanic patients had access to specialized epilepsy care compared to $57 \%$ of white patients.

Although access plays a major role in this surgical disparity, it is not the only reason minorities are receiving less care. Even when patients have access to specialized health centers, there is still a discrepancy in who undergoes surgery. Burneo et al. ${ }^{16}$ analyzed patients with epilepsy discharged from the University of Alabama Hospital and found that African American patients were less likely than non-Hispanic white patients to undergo surgical treatment (odds ratio 0.3). Even when controlling for confounding variables such as age, sex, education, socioeconomic status, medical insurance, and clinical condition, African American patients had 60\% decreased chance of undergoing surgery. All of the patients in the study were referred to an academic center, were eligible surgical candidates, and had adequate insurance coverage, yet there were still significantly fewer African American patients undergoing surgery. Similarly, Thompson et al. ${ }^{17}$ noted that African Americans, Asian/Pacific Islanders, and patients with 
Even when patients have access to specialized health centers there is still a discrepancy in who undergoes surgery.

limited English proficiency had significantly longer times to anterior temporal lobectomy surgery even when all patients underwent the full presurgical evaluation.

Studies specifically measuring improvement in surgical treatment after increased access to specialized centers are limited, although one can extrapolate information from broader medical studies. An example comes from the National Health Service in England, which has strengthened primary care to increase access, with only modest reductions in health care outcome inequalities. ${ }^{18}$ The authors argue that socioeconomic inequalities may also have to do with patient behaviors, social support networks, primary and secondary care provider behavior, and coordination of care between providers.

There is evidence to support access as the predominant reason why patients are less likely to undergo surgical evaluation, making it a more heavily weighted FACETS item.
However, once patients have reached the specialist's care, other factors are likely contributing to the reason why certain populations are not following through with surgical treatment.

\section{Communication barriers}

Language incompatibility between patient and physician can lead to patients experiencing difficulties communicating in English when sick. ${ }^{19}$ Compared to English-speaking USborn patients with epilepsy, Spanish-speaking patients in one study received significantly lower number of AEDs compared to the US-born patients ${ }^{20}$ This result remained true even though the 2 groups were of similar age, had epilepsy for the same number of years, and had similar seizure frequency. These data suggest that a complex procedure such as epilepsy surgery may suffer from the same predicament with language/communication.

Similar findings were demonstrated by a retrospective cohort study that revealed that regardless of race/ethnicity, patients with limited English proficiency who had mesial temporal sclerosis were significantly less likely to undergo anterior temporal lobectomy compared to English-speaking patients. ${ }^{6}$ The authors aptly cite research by Lasser et al., ${ }^{21}$ which demonstrated that colorectal cancer screening among nonEnglish-speaking patients was improved when language concordant patient navigators were used to discuss the screening options with patients. Currently no studies have been conducted that use similar methods in patients considering epilepsy surgery, making it an area open to study.

Figure Factors that influence patients' decision to undergo surgical treatment of epilepsy
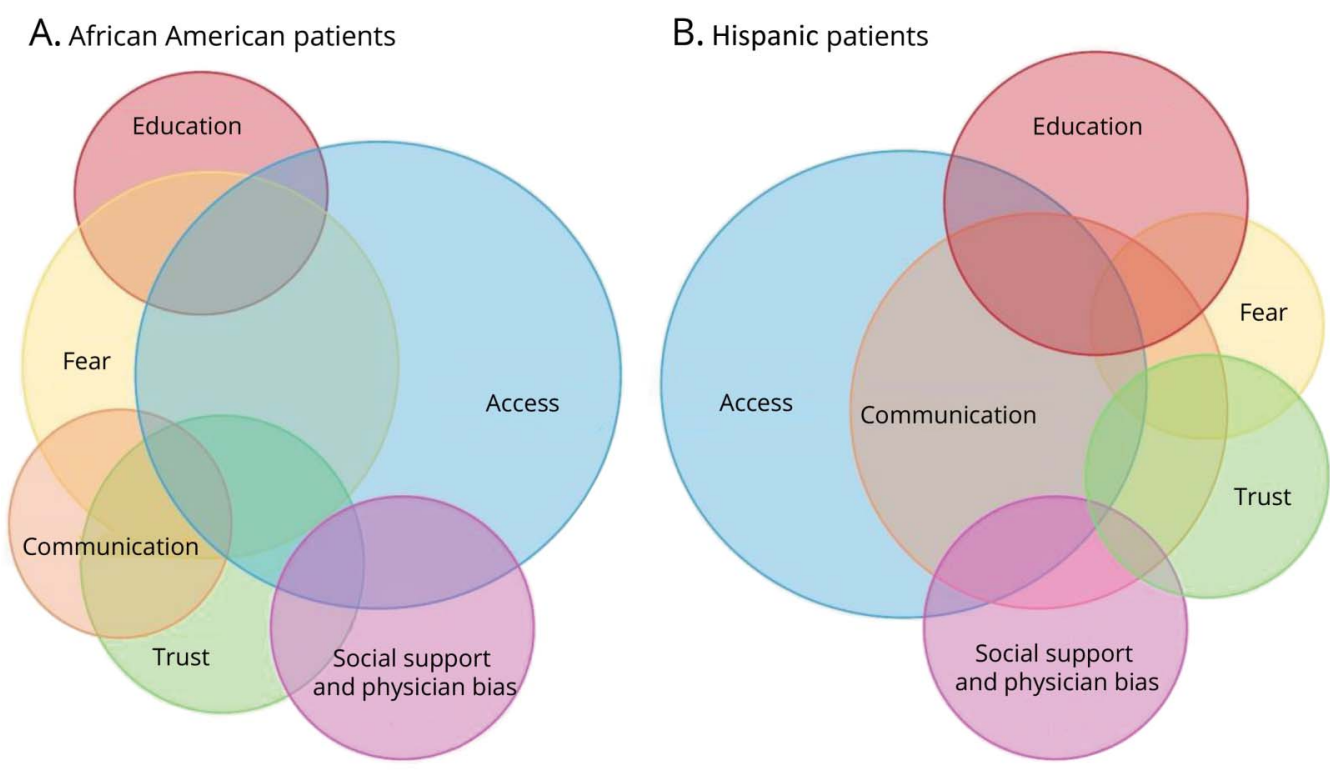

Visual depiction of the 6 proposed factors (FACETS) that influence patients' decision to undergo epilepsy surgery. (A) African American patients. (B) Hispanic patients. The size of each circle represents a proposed weight that each factor may have on a patient's decision (larger circle represents a more heavily weighted factor). The weight of each factor may vary depending on the patient's race, ethnicity, language, and cultural background (such as race/ethnicity, as depicted above). There is significant overlap between each of these factors as they are not mutually exclusive. Access appears to be the most studied and heavily weighted factor at this time, although further research needs to be conducted to elucidate the influence of the other factors on surgical treatment. 
Table Example of future measurement and interventions to help understand and decrease health disparities in epilepsy

\begin{tabular}{|c|c|c|}
\hline Factor & Examples of measurements & Examples of interventions \\
\hline Fear & Pain Anxiety Symptom Scale & $\begin{array}{l}\text { Patient education and empowerment } \\
\text { Provider cultural communication training } \\
\text { Patient testimonial } \\
\text { Community intervention }\end{array}$ \\
\hline Access & $\begin{array}{l}\text { Number of surgeries in level IV National Association } \\
\text { of Epilepsy Centers of referred minority groups vs } \\
\text { non-Hispanic white patients with similar insurance }\end{array}$ & $\begin{array}{l}\text { Provider bias education } \\
\text { Social worker } \\
\text { Community intervention } \\
\text { Telemedicine use }\end{array}$ \\
\hline Communication barriers & $\begin{array}{l}\text { Use of live language interpreters vs phone and } \\
\text { measure outcomes } \\
\text { Use of community volunteers to help improve } \\
\text { communication and measure of outcomes } \\
\text { Measuring physician time and interactions with } \\
\text { minority patients }\end{array}$ & $\begin{array}{l}\text { Provider bias education } \\
\text { Provider cultural competence and } \\
\text { communication education } \\
\text { Scribes } \\
\text { Patient-provider racial concordance }\end{array}$ \\
\hline Education & $\begin{array}{l}\text { Patient level of understanding of procedure after visit } \\
\text { and outcomes }\end{array}$ & $\begin{array}{l}\text { Visual aids and handouts to explain } \\
\text { procedures }\end{array}$ \\
\hline Trust & Distrust scale measurement and outcomes & $\begin{array}{l}\text { Community engagement } \\
\text { Patient testimonials }\end{array}$ \\
\hline Social support and physician bias & $\begin{array}{l}\text { Evaluation of postsurgery social resources and } \\
\text { number of surgeries } \\
\text { Physician education and knowledge about bias }\end{array}$ & $\begin{array}{l}\text { Postprocedure close follow-up with socia } \\
\text { worker and community contact } \\
\text { Pharmacist follow-up for medication } \\
\text { compliance and side effects } \\
\text { Provider bias education } \\
\text { Provider cultural competence } \\
\text { Close psychological support }\end{array}$ \\
\hline
\end{tabular}

Even when speaking the same language, physicians may still fail to get the message across in a manner that patients understand. McClelland et al. ${ }^{12}$ found that English-speaking African American patients were significantly less likely to undergo anterior temporal lobectomy surgery even when medically eligible. Communication skills were identified as a common physician-centered factor that can lead to such disparities. They support this notion by citing a study using postvisit surveys and audiotape analysis from physicianpatient interactions. African American patients had shorter visits with white physicians and physician verbal dominance was significantly higher in visits with African American patients than in visits with white patients (for both African American and white doctors). ${ }^{22}$

Despite the fact that communication is identified as a possible explanation for health disparities, more studies need to be conducted to parse out what can be done to improve communication between physician and patient.

\section{Education}

In general, people with epilepsy have lower education and health status compared to healthy adults. ${ }^{1,23}$ They are also less likely to complete secondary or postsecondary education compared to the general population. There is a slight yet significant difference in IQ between patients who undergo surgical treatment of epilepsy (IQ91.4, PIQ92.4) and those who opt out of treatment (IQ 89.5, PIQ 89.5) even though they are eligible for surgery. ${ }^{24}$ Education does not necessarily equate to IQ; it also involves the patient's health literacy. Furthermore, race/ethnicity may also influence a patient's understanding of his or her disease and treatment options. Bautista and Jain's ${ }^{25}$ study noted that African American patients had a greater mistrust for medications compared to white patients despite having similar education levels. The disparity in attitude towards medication might be related to knowledge about epilepsy. Pharmacist-centered patient educational programs improve patients' beliefs about medications, making this a viable option for improved care. This is a prime example of how the each of the FACETS items are not mutually exclusive in that mistrust and education both contributed in this case to a discrepancy in health care.

Patients of varying races/ethnicities may cope with their disease differently and seek different treatment options depending on their beliefs. For example, a survey among hundreds of Spanish-speaking adults in the United States found that $8 \%$ believe sins are a cause of seizure and $10 \%$ agree that exorcism is a viable remedy. ${ }^{26}$ Disparities in treatment for this patient population may be explained by a cultural understanding of the disease, further highlighting that education may be a more heavily weighted factor in treatment choice.

\section{Trust between patient and physician}

Trust is discussed in the previously mentioned research study from UCLA whereby African American patients described overall distrust in their health care providers. ${ }^{8}$ As further support of this idea, the authors mention the study by Collins et al. $^{27}$ that addressed racial differences in communication and decision-making with regards to invasive cardiac procedures. African American patients stressed the importance 
of trusting their health care provider before agreeing to invasive cardiac procedures. In addition, this same patient population complained of a lack of trust between themselves and health care providers.

Further support for mistrust among African American patients comes from the survey study by Bautista and Jain ${ }^{25}$ at a tertiary epilepsy center. Results indicated that African American patients had significantly more seizures and scored higher on the General Beliefs about Medicine Questionnaire, indicating a higher mistrust of medications.

Additional research needs to be done to determine how trust plays a role in epilepsy surgery with regards to various other patient populations. Physicians are not free of stigma and may also contribute to a lack of trust with patients.

\section{Social support}

This category encompasses factors such as income, family support, employment, and physician bias, as each of these factors can affect health care. Having epilepsy is associated with lower incomes, higher unemployment, and lower educational attainment. ${ }^{23}$ Lower socioeconomic status is also associated with poorer medication compliance, which could discourage this patient population from being epilepsy surgery candidates. ${ }^{28}$ People with epilepsy living in poverty experience "higher psychological distress" and "lower selfperceived health" compared to other groups. Home ownership is associated with a decreased risk of epilepsy and married patients are more likely than unmarried patients to undergo surgical treatment of epilepsy. ${ }^{24,29}$ A study from South Carolina revealed the hazard of mortality was higher among patients who lived in zip codes with an annual median income less than $\$ 36,000$. $^{22}$ The same study showed a weak yet significant higher mortality risk in African American patients with epilepsy compared to white patients.

Compared to US-born people with epilepsy, Hispanic patients have less comprehensive health insurance coverage, receive less AEDs, have higher depression scores, and receive less antidepressant medications. ${ }^{20}$ Authors from this study note the decreased quality of life in the Hispanic patients may be due to embarrassment associated with having seizures and the overall stigmatization about epilepsy within the culture.

\section{Physician bias}

There is growing evidence about the role of physician bias in health disparities. ${ }^{30-32}$ This has not been studied in epilepsy surgery but has been explored in other fields of medicine. A 2000 study asked physicians about their perception of patients who underwent coronary angiograms. ${ }^{30}$ Data from the survey after the procedure revealed African American patients were rated as less intelligent, less educated, and less compliant with medication recommendations compared to white patients. Another study used an Internet-based tool that presented physicians with clinical vignettes of patients of
TAKE-HOME POINTS

$\rightarrow$ The rate of epilepsy surgery is disproportionately lower among African American, Hispanic, and nonEnglish-speaking patients when compared to nonHispanic white patients in the United States.

$\rightarrow$ Factors that may be associated with this disparity include fear, access, communication barriers, education, trust between patient and provider, and social issues including physician bias. These can be organized under the acronym FACETS.

$\rightarrow$ The effect that each factor has on the disparity is discussed and may vary depending on race and cultural difference.

$\rightarrow$ FACETS can be used as framework to model the interactions, measure, and intervene in each factor based on racial and cultural differences.

various races in the emergency room with symptoms of acute coronary syndrome. ${ }^{31}$ Responders then answered questions using the Implicit Association Test, which measured implicit race preference and perceived notion of cooperativeness with regards to thrombolysis. Results revealed implicit stereotypes of African Americans as less cooperative with medical procedures, ultimately leading to more white patients being treated with thrombolysis compared to African American patients.

These studies highlight the need for further research that addresses physician bias among neurologists with regards to taking care of patients of various ethnicities and cultures as it can have devastating consequences on patient care.

\section{Moving forward}

FACETS in epilepsy surgery can be measured and intervened at the patient and provider level (table). Different groups should be studied separately to have a better understanding of the weight each factor may have (figure). The information obtained can then be used to establish quality metrics for physicians and institutions.

\section{Conclusion}

It is evident that health disparities in epilepsy surgery exist yet the exact reasoning why and the solutions to the gaps in care are understudied. Reasons have been posited but not proven, which is why the goal for the future is to systematically test each of the 6 FACETS of health disparities to discern which factor or factors causes the greatest obstacle for care. If physicians are more aware of the FACETS that stray people from surgical treatment, we hope that they will be able to better guide their patients' care. 


\section{Author contributions}

Cody L. Nathan: literature review, drafted the paper. Camilo Gutierrez: study concept, draft edits.

\section{Study funding}

No targeted funding reported.

\section{Disclosure}

The authors report no disclosures. Full disclosure form information provided by the authors is available with the full text of this article at Neurology.org/cp.

Received January 20, 2018. Accepted in final form April 27, 2018.

\section{References}

1. Centers for Disease Control and Prevention. Health disparities. Available at: cdc.gov/ healthyyouth/disparities/. Accessed June 14, 2018.

2. Hauser WA, Hesdorffer DC. Epilepsy: Frequency, Causes and Consequences. New York: Demos Press; 1990.

3. Centers for Disease Control and Prevention. Prevalence of self-reported epilepsy: United States, 1986-1990. MMWR Morb Mortal Wkly Rep 1994;43:810-811, 817-818.

4. Holden EW, Thanh Nguyen H, Grossman E, et al. Estimating prevalence, incidence, and disease-related mortality for patients with epilepsy in managed care organizations. Epilepsia 2005;46:311-319.

5. Szaflarski M, Szaflarski JP, Privitera MD, Ficker DM, Horner RD. Racial/ethnic disparities in the treatment of epilepsy: what do we know? What do we need to know? Epilepsy Behav 2006;9:243-264.

6. Betjemann JP, Thompson AC, Santos-Sanchez C, Garcia PA, Ivey SL. Distinguishing language and race disparities in epilepsy surgery. Epilepsy Behav 2013;28:444-449.

7. Schiltz NK, Koroukian SM, Singer ME, Love TE, Kaiboriboon K. Disparities in access to specialized epilepsy care. Epilepsy Res 2013;107:172-180.

8. Swarztrauber K, Dewar S, Engel J Jr. Patient attitudes about treatments for intractable epilepsy. Epilepsy Behav 2003;4:19-25.

9. Lavernia CJ, Alcerro JC, Rossi MD. Fear in arthroplasty surgery: the role of race. Clin Orthop Relat Res 2010;468:547-554.

10. Gillespie A, Hammer H, Bass S, et al. Attitudes towards living donor kidney transplantation among urban African American hemodialysis patients: a qualitative and quantitative analysis. J Health Care Poor Underserved 2015;26:852-872.

11. Englot DJ, Ouyang D, Garcia PA, Barbaro NM, Chang EF. Epilepsy surgery trends in the United States, 1990-2008. Neurology 2012;78:1200-1206.

12. McClelland S III, Guo H, Okuyemi KS. Racial disparities in the surgical management of intractable temporal lobe epilepsy in the United States: a population-based analysis. Arch Neurol 2010;67:577-583.
13. Sharma K, Kalakotia P, Henrya M, et al. Revisiting racial disparities in access to surgical management of drug-resistant temporal lobe epilepsy post implementation of Affordable Care Act. Clin Neurol Neurosurg 2017;158:82-89.

14. Begley CE, Basu R, Reynolds T, et al. Sociodemographic disparities in epilepsy care: results from the Houston/New York City health care use and outcomes study. Epilepsia 2009;50:1040-1050.

15. Fantaneanu TA, Hurwitz S, van Meurs K, Llewellyn N, O’Laughlin KN, Dworetzky BA. Racial differences in emergency department visits for seizures. Seizure 2016;40: $52-56$.

16. Burneo JG, Black L, Knowlton RC, Faught E, Morawetz R, Kuzniecky RI. Racial disparities in the use of surgical treatment for intractable temporal lobe epilepsy. Neurology 2005;64:50-54.

17. Thompson AC, Ivey SL, Lahiff M, Betjemann JP. Delays in time to surgery for minorities with temporal lobe epilepsy. Epilepsia 2014;55: 1339-1346.

18. Asaria M, Ali S, Doran T, et al. How a universal health system reduces inequalities: lessons from England. J Epidemiol Community Health 2016;70:637-643.

19. Documet PI, Sharma RK. Latinos' health care access: financial and cultural barriers. J Immigr Health 2004;6:5-13.

20. Myers L, Lancman M, Vazquez-Casals G, Bonafina M, Perrine K, Sabri J. Depression and quality of life in Spanish-speaking immigrant persons with epilepsy compared with those in English-speaking US-born persons with epilepsy. Epilepsy Behav 2015; 51:146-151.

21. Lasser KE, Murillo J, Lisboa S, et al. Colorectal cancer screening among ethnically diverse, low-income patients: a randomized controlled trial. Arch Intern Med 2011; 171:906-912.

22. Institute of Medicine. Unequal Treatment: Confronting Racial and Ethnic Disparities in Health Care. Washington, DC: The National Academies Press; 2003.

23. Burneo JG, Jette N, Theodore W, et al. Disparities in epilepsy: report of a systematic review by the North American Commission of the International League Against Epilepsy. Epilepsia 2009;50:2285-2295.

24. Berg AT, Vickrey BG, Langfitt JT, et al. The multicenter study of epilepsy surgery: recruitment and selection for surgery. Epilepsia 2003;44:1425-1433.

25. Bautista RE, Jain D. Detecting health disparities among Caucasians and African Americans with epilepsy. Epilepsy Behav 2011;20:52-56.

26. Sirven JI, Lopez RA, Vazquez B,Van Haverbeke P. Que es la epilepsia? Attitudes and knowledge of epilepsy by Spanish-speaking adults in the United States. Epilepsy Behav 2005;7:259-265.

27. Collins TC, Clark JA, Petersen LA, Kressin NR. Racial differences in how patients perceive physician communication regarding cardiac testing. Med Care 2002;40: I27-I34.

28. Szaflarski M. Social determinants of health in epilepsy. Epilepsy Behav 2014;41: 283-289.

29. Hesdorffer DC, Tian H, Anand K, et al. Socioeconomic status is a risk factor fo epilepsy in Icelandic adults but not in children. Epilepsia 2005;46:1297-1303.

30. van Ryn M, Burke J. The effect of patient race and socio-economic status on physicians' perceptions of patients. Soc Sci Med 2000;50:813-828.

31. Chapman EN, Kaatz A, Carnes M. Physicians and implicit bias: how doctors may unwittingly perpetuate health care disparities. J Gen Intern Med 2013;28: $1504-1510$

32. Green AR, Carney DR, Pallin DJ, et al. Implicit bias among physicians and its prediction of thrombolysis decisions for black and white patients. J Gen Intern Med 2007; 22:1231-1238.

\section{Subspecialty Alerts by E-mail!}

Customize your online journal experience by signing up for e-mail alerts related to your subspecialty or area of interest. Access this free service by clicking on the "My Alerts" link on the home page. An extensive list of subspecialties, methods, and study design choices will be available for you to choose from-allowing you priority alerts to cutting-edge research in your field! 